\title{
Maintenance treatment and shared care in lymphoblastic leukaemia
}

\author{
Judith M Chessells
}

Treatment of children with acute lymphoblastic leukaemia (ALL) and non-Hodgkin's lymphoma has become more intensive and more successful in recent years. The first few months from diagnosis are punctuated by repeated admissions to hospital for periods of intensive chemotherapy, and often by readmission for management of the resulting fever and neutropenia. However, over half of the treatment period for virtually all children with ALL and for many with non-Hodgkin's lymphoma of $T$ cell subtype is spent receiving oral drugs as an outpatient and during this phase of 'maintenance' treatment shared care between the tertiary centre and the local paediatric unit comes into its own.

How often should children have blood counts during maintenance treatment? What is the risk of infections and how should they be managed? What is the risk of chickenpox or measles? What advice should be given at the time of stopping treatment? These are some of the many questions asked by parents, paediatricians, and family doctors. In this article the author reviews her approach to these problems which arise during maintenance or continuing treatment.

\section{The role of continuing (maintenance) chemotherapy}

The term maintenance is an unfortunate one, and dates from the time when the aim of treatment of ALL was maintaining a remission to defer the inevitable relapse. A better term is perhaps continuing treatment and one which will be used here. It might be argued that, in the era of modern intensive therapy, children could be cured by a very intensive short term protocol without continuing treatment. However, all earlier attempts at such short sharp shock treatment in ALL, admittedly some 20 years ago, have resulted in an extremely high relapse rate. There have been few attempts to randomise duration of treatment more recently, but in one small trial children who received 18 months of treatment after induction and intensification had a higher relapse rate than those treated for two years. ${ }^{1}$ The medical and financial costs of rescue treatment after relapse are high, the success rate is uncertain ${ }^{2}$ and, as continuing treatment is relatively simple, it would seem that efforts to abandon it will have to proceed very cautiously, by attrition rather than by a sudden change of custom.

It may well be asked what maintenance treatment can actually do. ALL is the only form of childhood cancer where continuing treatment may be beneficial. There are various theories about how it works and this topic has been reviewed. ${ }^{3}$ One hypothesis is that treatment continues the systematic eradication of residual leukaemic cells set in motion by earlier induction and intensification. It seem surprising that such relatively mild treatment could have this effect. An alternative theory is that the immune system is stimulated by the long term oral drugs, a concept that seems unlikely, as daily continuing treatment is itself immunosuppressive. ${ }^{4}$ Lastly Gale and Butturini have postulated that as ALL is a disease of early $B$ cells which are normally programmed to death by apoptosis, continuing chemotherapy controls growth of the leukaemia clone so permitting cell death, an attractive although unproved concept. $^{3}$

\section{The choice of drugs in continuing treatment}

The standard combination of drugs given in this phase of treatment consists of daily mercaptopurine, weekly methotrexate, and prednisolone and vincristine monthly, a well known combination illustrated in the figure. Continuous daily treatment with mercaptopurine and methotrexate is superior to intermittent higher doses of these two drugs given every three weeks. ${ }^{4}$ There is little evidence that, after suitable periods of early intensification, more complicated regimens than this are of any additional benefit, and there can be no justification for the use of such regimens other than in a clinical trial.

\section{METHOTREXATE}

This was one of the original folate antagonists used in ALL and has been a mainstay of treatment ever since. A biological explanation for its efficacy may lie in the ability of lymphoblasts, particularly those of common ALL, to accumulate methotrexate, and the correlation of this ability with a better chance of long term survival. ${ }^{5}$ Methotrexate is usually given by mouth once weekly. Intramuscular 

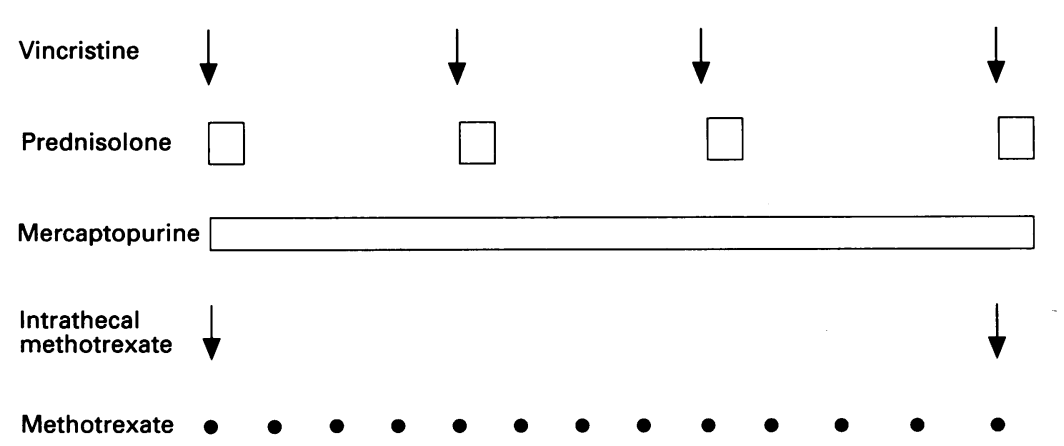

Co-trimoxazole $000 \quad 000000000000000000000000000000000000$

$\begin{array}{llllllllllllll}\text { Week } & 1 & 2 & 3 & 4 & 5 & 6 & 7 & 8 & 9 & 10 & 11 & 12 & 1\end{array}$

Flow diagram of the recurring cycle of continuing treatment.

methotrexate is not superior to oral, ${ }^{6}$ but is preferred in several north American protocols, ${ }^{7-9}$ presumably because this route ensures better compliance.

\section{MERCAPTOPURINE}

This is given by mouth daily and is activated to thioguanine nucleotides, cytotoxic metabolites which are incorporated into DNA. Children with higher levels of intracellular mercaptopurine metabolites are more likely to become neutropenic ${ }^{10}$ and have a better chance of long term remission. ${ }^{11}$ Boys appear to tolerate mercaptopurine better than girls, and this variable tolerance may be related to the still unexplained difference in prognosis between the sexes. ${ }^{12}$ Mercaptopurine has been a mainstay of continuing treatment for many years but it has recently been suggested that thioguanine may be more effective. ${ }^{13}{ }^{14}$ It is likely that the next Medical Research Council (MRC) ALL trial will include a randomised comparison of mercaptopurine and thioguanine and other collaborative groups are considering a similar comparison.

\section{PREDNISOLONE}

Prednisolone as a five day course and vincristine as a single intravenous injection are given monthly in addition to these drugs and may improve the chance of sustained remission without compromising dosage of mercaptopurine and methotrexate. ${ }^{15}$ The addition of other cytotoxic drugs to the combination of daily mercaptopurine and weekly methotrexate increased myelotoxicity and immunosuppression and did not improve long term survival. ${ }^{16}$

\section{CO-TRIMOXAZOLE}

This is normally given by mouth three times weekly to prevent Pneumocystis carinii pneumonitis. The adverse publicity given to cotrimoxazole in the media does periodically promote anxiety among families, but the drug is well tolerated in most patients, except for the association with neutropenia, and remains the most effective form of prophylaxis.

\section{Drug dose and compliance}

It is important that during continuing treatment drugs are given to the limits of tolerance as usually determined by the absolute neutrophil count. Children who receive the maximum tolerated doses of mercaptopurine and methotrexate drugs tend to have a lower relapse rate, 1217 and children with high erythrocyte concentrations of drug metabolites have a lower risk of relapse. ${ }^{18}$ There has perhaps been insufficient attention paid to the issue of compliance during treatment, but there seems no reason to believe that this should not be a problem in ALL as in other conditions, and evidence of non-compliance with both mercaptopurine and steroids has been documented. 1920

\section{Planning continuing (maintenance) chemotherapy}

Parents are bombarded with facts at the time of diagnosis of childhood leukaemia and it is important to recognise that all information given at the time of diagnosis needs to be repeated and reinforced. A natural time to discuss continuing treatment in detail is after completion of central nervous system directed treatment, whether this has comprised cranial irradiation, intravenous methotrexate, or a course of weekly intrathecal methotrexate injections. At this stage it is worth spending time with parents and patients if appropriate going through the continuing treatment diagram (figure), stressing the importance of adequate drug doses and adjustments according to the blood count. A comparison can be drawn with adjustments of insulin dose made in childhood diabetes. It is our practice in clinic to give parents the follow up forms, together with a booklet we have prepared about continuing chemotherapy, which contains instructions on completion of the forms, details about drug dose adjustment, and appendices containing tables of the appropriate full and reduced doses of drugs according to surface area and the number of tablets needed to provide appropriate doses of drugs. Most parents are anxious to learn and to contribute as much as possible to treatment and, provided the booklet and instructions are also sent to the shared care centre, this system seems to work well. Drugs are started at the recommended top dose based on surface area and parents are warned that this is merely an empirical start dose, that the right dose is the maximum one tolerated by the individual patient, that one or more episodes of neutropenia are inevitable and do not herald relapse of leukaemia.

It is important to ensure a follow up discussion, usually at the shared care centre to make sure that these points have been understood. Calculation of surface area every three months provides an opportunity for reviewing the drug dose during the preceding cycle and ensures that the height is regularly recorded to facilitate updating of the growth chart, which should be maintained in every child receiving chemotherapy. This regular review of drug doses is 
essential throughout treatment and, unfortunately, blood counts are essential every one to two weeks to ensure that children receive the maximum tolerated dose of drugs.

\section{Duration of continuing chemotherapy}

There have been a large number of trials involving randomisation of the duration of treatment. The most recent one conducted by the MRC was UKALL VIII, ${ }^{21}$ where a third year of treatment provided no benefit. At present treatment in the $\mathrm{UK}$ is continued for two years or rather 100 weeks from diagnosis. There is little current enthusiasm for further randomised trials of duration of treatment and much eagerness to explore the applicability of testing for minimal residual disease, a topic which has been extensively reviewed..$^{22} \mathrm{~A}$ crude morphological examination of the bone marrow detects at best $3-5 \%$ of leukaemic cells. The use of combinations of monoclonal antibodies can detect 1 in 10000 cells and modern techniques of molecular biology are in theory even more sensitive than this. There have been a number of published reports showing that the presence of minimal residual disease may herald impending relapse. However, a number of uncertainties remain in this field; these are both methodological and concern the significance of finding minimal residual disease at various time points from diagnosis. Moreover, leukaemic cells detected in this way may not necessarily have the potential for replication. At present therefore it is clear that detection of minimal residual disease is feasible but its practical impact needs to be assessed in large scale prospective trials, planned both in the UK and many other countries.

\section{Management of neutropenia}

During continuing treatment the absolute neutrophil count should be between $1 \cdot 0-2 \cdot 0 \times 10^{9} / 1$ and so virtually all children who are receiving proper drug doses will become neutropenic sooner or later, that is with absolute counts of under $1.0 \times 10^{9} / 1$. It is usual to halve the doses of both mercaptopurine and methotrexate if the absolute neutrophil count falls below 1.0 and stop both drugs if it falls below 0.5 , but vincristine and prednisolone can be continued as scheduled since they are not myelotoxic. Although protocols also dictate drug dose modifications for thrombocytopenia, the count rarely falls below $100 \times 10^{9} / \mathrm{l}$. There is no point in making these modifications in relation to blood counts performed more than once weekly as the count always takes at least one, and often two, weeks to recover. If neutropenia persists despite two weeks without treatment, then it is usual to stop co-trimoxazole. There are some children who become neutropenic every time cotrimoxazole is resumed and in these cases we normally stop it in the interest of maintaining reasonable chemotherapy doses. Our own practice is not to substitute pentamidine inhalation routinely but to reserve it for very high risk situations. It is necessary, however, in these circumstances, to maintain a high threshold of suspicion for $P$ carinii pneumonitis and be prepared to treat empirically with high dose co-trimoxazole.

Although conventional wisdom dictates that a bone marrow should be done if neutropenia persists for more than two weeks after drugs have been stopped this is virtually never symptomatic of relapse, and naturally raises a high level of anxiety. If co-trimoxazole is stopped the count will virtually always recover within a week or 10 days. If children repeatedly drop their counts when full drug doses are resumed it is better to settle for sustained chemotherapy, albeit at a lower dose, than a continuing cycle of interruptions of treatment.

There is no reason to keep children off school, away from playgroup, or even worse in hospital if they are neutropenic during continuing treatment. The drugs used in continuing treatment do not cause acute gut toxicity and thus fever in association with neutropenia is rare, and will not be prevented by social isolation.

\section{Children who are never neutropenic}

There are some children who, while prescribed maximum drug doses according to protocol, or even higher doses, never become neutropenic. It is very important to check that there is no misunderstanding about giving the drugs. There is evidence that poor compliance may be a factor here and it is important to consider this, and perhaps measure thiopurine metabolites. Boys tolerate higher doses of mercaptopurine than girls and become neutropenic less readily; this may be a factor in their higher relapse rate. ${ }^{12}$ It is important to increase the dose of 6-mercaptopurine consistently until the leucocyte count drops.

\section{Infections during continuing (maintenance) treatment}

The greatest cause of anxiety, and of telephone calls to the clinic, is the concern about serious infections during treatment and without active support and encouragement to do otherwise there is a real danger of children missing school or playgroup. There is no doubt that continuing treatment is immunosuppressive, and tragically up to $5 \%$ of children may die from intercurrent infection during treatment. ${ }^{23}$ However, many of these deaths are avoidable, and are due to a limited number of infections discussed below. There is little evidence that children with ALL are more likely to get common mild viral infections than other children, and, with the exceptions mentioned below, any intercurrent infections should be managed in the normal way.

\section{MEASLES}

This is potentially the most serious infection during treatment as measles in the unimmunised child is untreatable and leads all too often, without a characteristic rash, to progressive 
interstitial pneumonia and death, or occasionally to encephalitis. The diagnosis is confirmed by immunofluorescent examination of a sample of nasopharyngeal aspirate. The high level of immunisation in the population has, mercifully, rendered this problem less common. We recommend that siblings and family contacts are immunised, as there is no evidence that the attenuated vaccine can be transmitted. The unimmunised child should avoid contact with measles at all costs, and in the event of contact should receive broad spectrum immune globulin.

\section{VARICELLA ZOSTER}

Fear of chickenpox is perhaps the commonest cause of school absence. Children who have not had chickenpox are susceptible to infection and zoster immune globulin (ZIG) should be given within 72 hours of contact. ZIG is effective for three to four weeks and rather than keep children off school we tend to repeat the injection when chickenpox is rife. The incubation period of chickenpox can be prolonged after ZIG to up to four weeks, but the resulting infection is usually mild. The dreaded complications of dissemination with pneumonia, or encephalitis can be avoided by prompt treatment with intravenous acyclovir.

\section{HERPES SIMPLEX}

Some children suffer from recurrent cold sores, and may rarely develop disseminated herpes, so that persistent herpes infections should be treated with oral acyclovir.

\section{PNEUMONIA}

$P$ carinii pneumonitis is very rare in children who have been taking co-trimoxazole as prescribed. The diagnosis can almost be made in clinic when a child who is not on co-trimoxazole presents with fever, cough and tachypnoea, but has no abnormal signs on auscultation and has a radiograph that shows interstitial pneumonia. The most common alternative diagnosis is mycoplasma pneumonitis, but this is usually associated with crepitations. The standard approach in this situation is to start treatment with high dose co-trimoxazole and erythromycin. A child who does not respond within two days to such treatment, or who deteriorates, should be referred back to the centre. If preliminary investigations are negative our usual next approach is to perform bronchoalveolar lavage and add in steroids and sometimes surfactant. Other causes of an interstitial pneumonia such as cytomegalovirus, candida, and respiratory viruses are relatively rare during continuing treatment, although of course common after bone marrow transplantation.

\section{CYTOMEGALOVIRUS}

This scourge of the bone marrow transplant patient is uncommon during continuing treatment, but may rarely cause fever, hepatosplenomegaly, intolerance of chemotherapy or pneumonitis, or retinitis. Treatment with gancyclovir is indicated if the diagnosis is confirmed.

\section{PARVOVIRUS}

This is a rare cause of chronic anaemia in immunosuppressed children including those with ALL during chemotherapy. ${ }^{24}$ If confirmed serologically it should be treated with high dose intravenous immunoglobulin.

\section{INFECTION IN IMPLANTED DEVICES}

Most children with ALL now have Hickman catheters or Portacaths inserted to facilitate intravenous access and unfortunately these may become infected. Clearly persistent fever or rigors on accessing the line should be investigated by peripheral and line cultures. If attempts to eradicate the infection with intravenous antibiotics are unsuccessful, or infection recurs, then the line must be removed.

\section{Other complications during treatment \\ NAUSEA AND VOMITING}

Some children become nauseated particularly when drugs are given in the mornings and it may be necessary to give them in the evening instead. Persistent, early morning vomiting, especially when accompanied by headache, may of course be symptomatic of central nervous system relapse.

\section{JAUNDICE AND ABNORMAL LIVER FUNCTION} TESTS

Transient abnormalities of liver function are common during continuing treatment and should not cause undue concern unless accompanied by jaundice. If abnormalities persist then investigation for hepatitis and cytomegalovirus is warranted.

\section{DIARRHOEA}

There are numerous causes of diarrhoea during treatment and in some children methotrexate may lead to enteritis. ${ }^{25}$ It is important to exclude infective causes of diarrhoea such as cryptosporidium.

\section{Bone marrows and lumbar punctures}

Routine bone marrows during and after stopping treatment do not influence prognosis and have been abandoned in most clinics including ours. It is, however, conceivable that the facility to monitor minimal residual disease may lead to reintroduction of marrows at some stages during treatment. By contrast, now that cranial irradiation has been abandoned for many children the majority now have regular lumbar punctures during treatment. It is important that central nervous system relapse is well documented as reactive mononuclear 
cells are common during regular intrathecal chemotherapy and these can be extremely difficult to differentiate from leukaemic blast cells.

\section{Immunisation policy}

Live vaccines should not be given during treatment. They are not effective and may prove harmful. We recommend that children are revaccinated at six months after stopping treatment with diphtheria, polio, and tetanus vaccines. If a child was fully immunised before diagnosis of leukaemia then only boosters are indicated. Measles, mumps, and rubella vaccine is given one year from completion of treatment. Full immunisation should be given to siblings and close friends; the only difference in the standard policy is that killed polio is recommended rather than live vaccines because of the risk of transmission of attenuated vaccine.

\section{Stopping treatment}

Stopping treatment is an extremely stressful time for families. Although the routine of treatment is burdensome there is a feeling that the treatment has kept the child well and the prospect of stopping the treatment reawakens all the natural anxieties about the future. It is important to recognise this and to give parents time for questions and discussion, backed up by an appointment at the tertiary centre.

We have found that giving parents a leaflet one to two months before the projected time of stopping, acts as a reminder for questions or problems. It is important to explain to the child that, although treatment is now stopping, follow up with blood counts and physical examinations will continue and that there can be no promises that treatment may not have to be resumed. A bone marrow and lumbar puncture should be performed at this time to document remission; testicular biopsies have not proved helpful in predicting testicular relapse and most centres have long since abandoned them.

\section{Follow up off treatment}

The risk of relapse after stopping treatment is maximal in the first two years but continues, albeit at a lower rate, for at least 10 years. Retrospective analysis of bone marrow samples in patients who have experienced such late relapses has indicated that these are indeed recurrences of the original leukaemia rather than development of a second malignancy. ${ }^{26}$ Relapse may be suspected on routine blood count, which we recommend is performed monthly for at least 18 months after stopping treatment, increasing the time interval thereafter. The testis remains an important site of relapse in boys and any untoward swelling of the testis should be reported and biopsied as soon as possible; despite the fact that testicular relapse is associated with subclinical disease elsewhere isolated relapse with no obvious bone marrow infiltration remains curable by conventional treatment. Central nervous system relapse at this stage is rare, but should be suspected in the event of the symptoms mentioned previously. The only other site occurring with any frequency is relapse in the iris which presents with apparent iridocyclitis; responding temporarily to steroids and confirmed on biopsy of the iris or examination for leukaemic cells in the anterior chamber.

\section{Planning for long term follow up}

Maintaining the medical records of all children with chronic disease is a challenge and leukaemia is no exception. A useful exercise at the time of stopping treatment, and one which saves trouble later, is preparation of a treatment summary documenting the protocol, doses, and scheduling of drugs and noting potential late effects. The summary can also give guidance for follow up: for example monitoring with echocardiography after anthracyclines, emphasis on checking growth and puberty after cranial irradiation. It is obviously important to explain this to patients and parents at an appropriate time. Once the child is past the time of maximum risk of relapse, the challenge is to maintain sensitive and appropriate follow up while ensuring that they do not feel a perpetual patient. I would like to thank my colleagues, in particular, Dr Ian Hann
for critical review of this manuscript, Judith Russell for typing, for critical review of this manuscript, Judith Russell for typing, port. Copies of our leaflets on 'Continuing Chemotherapy' and port. Copies of our leaflets on 'Continuing Chemotherathe
'Stopping Treatment' are available from the author.

1 Riehm H, Gadner H, Henze G, et al. Results and significance of six randomized trials in four consecutive ALL BFM studies. Haematology and Blood Transfusion 1990; 33: 439-50.

2 Chessells JM, Leiper AD, Richards SM. A second course of treatment for childhood acute lymphoblastic leukaemia: long-term follow-up is needed to assess results. $\mathrm{Br} \mathscr{F}$ Haematol 1994; 86: 48-54.

3 Gale RP, Butturini A. Maintenance chemotherapy and cure of childhood acute lymphoblastic leukaemia. Lancet 1991; 338: 1315-8.

4 Medical Research Council. Medical Research Council Leukaemia Trial-UKALL V: an attempt to reduce the immunosuppressive effects of therapy in childhood acute lymphoblastic leukemia. 7 Clin Oncol 1986; 4: 1758-64.

5 Whitehead VM, Rosenblatt DS, Vuchich M-J, et al. Accumulation of methotrexate and methotrexate polygluAccumulation of methotrexate and methotrexate polyglu-
tamates in lymphoblasts at diagnosis of childhood acute lymphoblastic leukemia: a pilot prognostic factor analysis. Blood 1990; 76: 44-9.

6 Chessells JM, Leiper AD, Tiedemann K, Hardisty RM, Richards S. Oral methotrexate is as effective as intramuscular in continuing (maintenance) therapy of acute lymphoblastic leukaemia. Arch Dis Child 1987; 62: 172-6.

7 Rivera GK, Raimondi SC, Hancock ML, et al. Improved outcome in childhood acute lymphoblastic leukaemia with reinforced early treatment and rotational combination chemotherapy. Lancet 1991; 337: 61-6.

8 Camitta B, Leventhal B, Lauer S, et al. Intermediate-dose intravenous methotrexate and mercaptopurine therapy for non-T, non-B acute lymphobytic leukemia of childhood: non-T, non-B acute lymphobytic leukemia of childhood: a pediatric

9 Clavell LA, Gelber RD, Cohen HJ, et al. Four-agent induction and intensive asparaginase therapy for treatment of tion and intensive asparaginase therapy for treatment of
childhood acute lymphoblastic leukemia. $N$ Engl f Med childhood acute lym

10 Lennard L, Lilleyman JS. Variable mercaptopurine metabolism and treatment outcome in childhood lymphoblastic leukemia. F Clin Oncol 1989; 7: 1816-23.

11 Lilleyman JS, Lennard L. Mercaptopurine metabolism and risk of relapse in childhood lymphoblastic leukaemia. Lancet 1994; 343: 1188-90.

12 Hale JP, Lilleyman JS. Importance of 6-mercaptopurine dose in lymphoblastic leukaemia. Arch Dis Child 1991; 66: $462-6$

13 Lennard L, Davies HA, Lilleyman JS. Is 6-thioguanine more appropriate than 6-mercaptopurine for children with acute lymphoblastic leukaemia? $B r 7$ Cancer 1993; 68: 186-90.

4 Adamson PC, Poplack DG, Balis FM. The cytotoxicity of thioguanine vs mercaptopurine in acute lymphoblastic leukemia. Leuk Res 1994; 18: 805-10. 
15 Bleyer WA, Sather HN, Nickerson $\mathrm{HJ}$, et al. Monthly pulses of vincristine and prednisone prevent bone marrow and testicular relapse in low-risk childhood acute lymphoblastic leukemia: a report of the CCG-161 study by the Children's Cancer Study Group. $₹$ Clin Oncol 1991; 9: 1012-21.

16 Aur RJA, Simone JV, Verzosa MS, et al. Childhood acute lymphocytic leukemia - study VIII. Cancer 1978; 42: lymphocy

17 Pearson ADJ, Amineddine HA, Yule M, et al. The influence of serum methotrexate concentrations and drug dosage on outcome in childhood acute lymphoblastic leukaemia Br F Cancer 1991; 64: 169-73.

18 Schmiegelow K, Schroder H, Gustafsson G, et al. Risk of relapse in childhood acute lymphoblastic leukaemia is related to RBC methotrexate and mercaptopurine metabolites during maintenance chemotherapy. $f$ Clin Oncol 1995; 13: 345-51. 19 Davies HA, Lennard L, Lilleyman JS. Variable mercaptoof non-compliance? BMF 1993; 306: 1239-40.

20 Peeters M, Koren G, Jakubovicz D, et al. Physician compliance and relapse rates of acute lymphoblastic leukemia in children. Clin Pharmacol Ther 1988; 43: 228-32.

21 Eden OB, Lilleyman JS, Richards S, et al. Results of Medical Research Council Childhood leukaemia tria UKALL VIII (report to the Medical Research Council on behalf of the working party on leukaemia in childhood). Br f Haematol 1991; 78: 187-97.

22 Campana D, Pui C- $\mathrm{H}$. Detection of minimal residual disease in acute leukemia: methodologic advances and disease in acute leukemia: methodologic ad

23 Atra A, Richards SM, Chessells JM. Remission death in acute lymphoblastic leukaemia: a changing pattern. Arch Dis Child 1993; 69: 550-4.

24 Kurtzman GJ, Cohen B, Meyers P, et al. Persistent B19 parvovirus infection as a cause of severe chronic anaemia in children with acute lymphocytic leukaemia. Lancet 1988; ii: 1159-62.

25 Craft AW, Kay HEM, Lawson DN, et al. Methotrexateinduced malabsorption in children with acute lymphoblastic leukaemia. BMF 1977; ii: 1511-2.

26 Potter MN, Steward CG, Oakhill A. The significance of detection of minimal residual disease in childhood acute lymphoblastic leukaemia. Br f Haematol 1993; 83: 412-8. 